\title{
Variétés faiblement spéciales à courbes entières dégénérées
}

\author{
Frédéric Campana et Mihai Păun
}

\begin{abstract}
A complex projective manifold is said to be weakly special if none of its finite étale covers has a dominant rational map to a positive-dimensional manifold of general type. Weakly special manifolds are conjectured by Harris and Tschinkel (Rational points on quartics, Duke Math. J. 104 (2000), 477-500) to be potentially dense if defined over a number field. In a previous paper by F. Campana (Special varieties, orbifolds and classification theory, Ann. Inst. Fourier (Grenoble) 54 (2004), 499-665), the stronger notion of special (complex projective) manifolds was introduced. These are conjectured in (9.20) of that work to be exactly the potentially dense manifolds (when defined over a number field). The two notions of specialness and weak-specialness coincide up to dimension 2, but differ from dimension 3 on, as shown by examples $X$ constructed by Bogomolov and Tschinkel (Special elliptic fibrations, in Proc. Fanu Conf., Torino, 2003, ed. A. Conte), answering a question raised in Campana's paper. These examples should thus be potentially dense, according to the first conjecture, but should not be so, according to the second conjecture. The present techniques of arithmetic geometry do not seem to be able to decide between these two conflicting conjectures. So, instead of this, we investigate the more tractable hyperbolic aspects of the examples of Bogomolov and Tschinkel, and show that for certain manifolds $X$, the behaviour of entire transcendental curves is that given in Conjecture 9.16, p. 618 of Campana's above-mentioned work. More precisely, the image of any holomorphic map from $\mathbb{C}$ to $X$ is contained either in some fibre of the unique elliptic fibration on $X$, or in some fixed divisor of $X$. This is consistent with the standard links (Lang and Vojta for varieties of general type, the first author's previous work in general) expected between the hyperbolic and arithmetic behaviour of projective varieties in cases where the second conjecture (stated by Campana) holds. Conversely, if the conjecture stated by Harris and Tschinkel is true, these expected links between arithmetics and complex hyperbolicity should fail to hold on these examples already.
\end{abstract}

\section{Introduction}

Soit $X$ une variété projective complexe lisse et connexe. Une fibration $\varphi: X \rightarrow Y$ est une application méromorphe dominante à fibres connexes, avec $Y$ lisse.

On dit que $X$ est faiblement-spéciale (ou $f$-spéciale) s'il n'existe pas de revêtement étale fini $u: X^{\prime} \rightarrow X$ et de fibration $\varphi: X^{\prime} \rightarrow Y$, où $Y$ est une variété de type général de dimension $p>0$ (voir [Cam04, 9.26, p. 621] pour cette notion).

Received 7 December 2005, accepted in final form 16 June 2006.

2000 Mathematics Subject Classification 14C30, 14G05, 14J27, 14J30, 32A22, 32J15, 32J17, 32 J25.

Keywords: complex hyperbolicity, Nevanlinna theory, orbifold.

This journal is (C) Foundation Compositio Mathematica 2007. 


\section{F. Campana et M. PăUN}

On dit que $X$ est spéciale s'il n'existe pas de fibration de type général $\varphi: X \rightarrow Y$, avec $\operatorname{dim}(Y):=$ $p>0$. Voir plus de détails dans le $\S 1$ ci-dessous.

La notion de fibration de type général est définie dans [Cam04, 2.1, p. 527]. Elle signifie que la base orbifolde de $\varphi$ déduite de ses fibres multiples est de type général pour tout modèle biméromorphe de $\varphi$. (Si $\varphi$ n'a pas de fibre multiple en codimension $1, \varphi$ est de type général si et seulement si $Y$ l'est). Voir $\S 1$ ci-dessous pour ces notions.

Une variété spéciale est $f$-spéciale (voir [Cam04, 1.8, 9.27]). Réciproquement, les courbes et surfaces spéciales sont $f$-spéciales. Des exemples de variétés faiblement spéciales non-spéciales de dimension 3 sont cependant construites par Bogomolov et Tschinkel dans [BT03], répondant ainsi affirmativement à une question posée dans [Cam04]. La construction de [BT03] est rappelée dans $\S 1$ ci-dessous. Les exemples de Bogomolov-Tschinkel sont simplement connexes et munis d'une unique fibration elliptique $\varphi: X \rightarrow B$, où $B$ est une surface avec $\kappa(B)=1$; cette fibration $\varphi$ est cependant de type général, car elle a des fibres multiples de multiplicité $m \geqslant 2$ au-dessus d'une courbe $D$ telle que $\kappa\left(B, K_{B}+(1-1 / m) . D\right)=2$. Mais ces fibres multiples ne peuvent être éliminées par un revêtement étale de $X$, qui est simplement connexe.

Pour $X$ définie sur un corps de nombres $k$, on dispose de 2 conjectures concernant la densité potentielle de $X$ (i.e. le fait que $X(K)$ soit Zariski dense dans $X$ pour une extension finie assez grande $K / k)$.

A. Il est conjecturé dans [HT00] (conjecture attribuée à D. Abramovich et J. L. Colliot-Thélène) que $X$ est potentiellement dense si et seulement si $X$ est faiblement spéciale. Cette conjecture affirme donc que les $X$ de [BT03] sont potentiellement denses (on peut en définir sur des corps de nombres).

B. Il est indépendamment conjecturé dans [Cam04, 9.20, p. 619] que $X$ est potentiellement dense si et seulement si $X$ est spéciale. Cette conjecture affirme donc que les $X$ de [BT03] ne sont pas potentiellement denses.

Les techniques actuelles de géométrie arithmétique ne semblent pas pouvoir décider entre ces deux affirmations contradictoires. On va donc considérer ici les propriétés, plus accessibles, d'hyperbolicité complexe des exemples de Bogomolov-Tschinkel. Ces propriétés sont conjecturalement (Lang et Vojta pour les variétés de type général, [Cam04] pour les variétés arbitraires) de nature géométrique, et équivalentes aux propriétés qualitatives de distribution des points $K$-rationnels, après extension assez grande $K$ du corps de définition.

Nous allons montrer que, pour certains des exemples de [BT03], les courbes entières transcendantes $h: \mathbb{C} \rightarrow X$ tracées sur $X$ sont algébriquement dégénérées et que leur image est, soit contenue dans un diviseur $Z$ fixé de $X$, soit contenue dans une fibre de $\varphi: X \rightarrow B$.

Si la conjecture A est vraie, cette propriété montre que les correspondances conjecturales attendues entre hyperbolicité et arithmétique sont fausses. Par contre, cette propriété d'hyperbolicité est exactement celle conjecturée dans [Cam04], et est compatible avec la conjecture B et les correspondances attendues entre hyperbolicité et arithmétique.

La conjecture $H$ de [Cam04, 9.2, p. 614] affirme en effet que $X$ est spéciale si et seulement si sa pseudométrique de Kobayashi est identiquement nulle, et dans le cas non-spécial des $X$ précédentes, que la pseudométrique de Kobayashi de $X$ est l'image réciproque par $\varphi$ d'une pseudométrique sur $B$, qui est une métrique sur un ouvert de Zariski dense de $B$. (Ceci peut être vu comme une version orbifolde de la Conjecture hyperbolique de S. Lang pour les surfaces de type général.) En particulier, pour les $X$ de Bogomolov-Tschinkel, la projection par $\varphi$ des courbes entières tracées sur $X$ doit être, soit réduite à un point, soit contenue dans un ensemble algébrique strict de $B$, qui est donc une réunion finie de courbes rationnelles ou elliptiques. C'est en effet ce que nous démontrons. 


\section{VARIÉTÉS FAIBLEMENT SPÉCIALES À COURBES ENTIÈRES DÉGÉNÉRÉES}

La méthode de démonstration consiste à exploiter le fait que la base orbifolde $(B / \Delta)$ de $\varphi$ est de type général, avec $\Delta:=(1-1 / m)$. $D$. Si le diviseur $D$ de $B$ était divisible par $m$ dans $\operatorname{Pic}(B)$, cette orbifolde serait revêtue de manière étale au sens orbifolde par un revêtement cyclique $\tau: B^{\prime} \rightarrow B$ de degré $m$ de $B$ ramifiant à l'ordre $m$ exactement le long de $D, B^{\prime}$ étant une surface de type général, puisque $K_{B^{\prime}}=\tau^{*}\left(K_{B}+\Delta\right)$. Le changement de base normalisé $B^{\prime} \rightarrow B$ fournirait alors $\varphi^{\prime}: X^{\prime}:=X \times_{B} B^{\prime} \rightarrow B^{\prime}$, avec $X^{\prime}$ étale sur $X$, donc tel que les courbes rationnelles ou elliptiques (respectivement les courbes entières) tracées sur $X$ se relèvent à $X^{\prime}$. La question se ramènerait donc, dans ce cas, après projection sur $B^{\prime}$ par $\varphi$, à montrer la finitude des courbes rationnelles ou elliptiques sur $B^{\prime}$, ainsi que la dégénérescence algébrique des courbes entières tracées sur $B^{\prime}$. $\mathrm{Si}$, de plus, le couple $(B, D)$ était choisi tel que $\left(c_{1}^{2}-c_{2}\right)\left(B^{\prime}\right)>0$, on déduirait de [Bog77] (respectivement $[\mathrm{McQ} 98]$ ) la première (respectivement la seconde) propriété.

Cependant, le fait que $X$ soit $f$-spéciale est exactement ce qui interdit à cette approche de fonctionner telle quelle (observer que nous pouvons contourner la difficulté dans le $\S 3$ par des choix adéquats pour la première propriété, mais non pour la seconde). La propriété cruciale utilisée (et qui traduit l'existence de fibres multiples de $\varphi$ ) est la suivante : si $h: \mathbb{C} \rightarrow X$ est une courbe entière sur $X$, alors sa projection $\varphi \circ h: \mathbb{C} \rightarrow B$ est $m$-tangente à $D$, ce qui signifie qu'en chaque point d'intersection de l'image de cette courbe avec $D$, l'ordre de contact est divisible par $m$ (on pourrait se contenter de au moins $m$, qui est la bonne notion). On montre alors que les courbes entières de $B$ qui sont $m$-tangentes à $D$ sont algébriquement dégénérées si $\left(c_{1}^{2}-c_{2}\right)(B / \Delta)>0$ (dans un sens adéquat). La méthode consiste à définir et montrer l'existence de sections non nulles de $\left[\operatorname{Sym}^{N}\right]\left(\Omega_{(B / \Delta)}^{1}\right) \otimes A^{-1}$, $A$ ample sur $B$, pour $N$ assez grand, sous ces hypothèses. Le symbole $\left[\mathrm{Sym}^{N}\right]$ désigne la partie entière du faisceau considéré (qui n'a de sens que localement, une fois une racine $m$-ième d'un équation locale de $D$ ayant été choisie). Des arguments classiques de théorie de Nevanlinna, complétés par des résultats récents concernant les courants définis par les courbes entières tracées sur les surfaces [McQ98, Bru99, Pau03] s'adaptent alors à notre contexte orbifolde et permettent de conclure. Voir le $\S 4$ pour les détails.

Nous remercions L. Caporaso pour nous avoir indiqué de nombreuses imperfections dans la première version de ce texte.

\section{Variétés spéciales}

Nous rappelons brièvement les notions introduites dans [Cam04], et qui jouent un rôle pour la compréhension de la suite.

Soit $X$ une variété projective complexe lisse et connexe. Une fibration (régulière) $f: X \rightarrow Y$ est un morphisme de $X$ sur une variété projective lisse $Y$, à fibres connexes. Une modification de $f$ est une fibration $f^{\prime}: X^{\prime} \rightarrow Y^{\prime}$ avec $u: X^{\prime} \rightarrow X, v: Y^{\prime} \rightarrow Y$ des modifications propres, et telles que $f u=v f^{\prime}$.

La base orbifolde de $f$, notée $\Delta(f):=\sum_{D}(1-1 / m(f, D)) . D$ est le $\mathbb{Q}$-diviseur (effectif, fini) sur $Y$ défini comme suit : $D$ varie parmi tous les diviseurs effectifs irréductibles de $Y$, et pour un tel $D, m(f, D) \geqslant 1$ est l'entier défini par $m(f, D):=\inf \left\{m_{j}, j \in J(D, f)\right\}$. On a utilisé les notations suivantes : $f^{*}(D)=\sum_{j \in J(D, f)} m_{j} . E_{j}+R$, où $R$ est un diviseur de $X$ tel que $f(R)$ soit de codimension au moins 2 dans $Y$, et les $E_{j}$ sont les diviseurs irréductibles distincts de $X$ tels que $f\left(E_{j}\right)=D, \forall j$.

On définit alors :

(1) le fibré canonique de $(Y / \Delta(f)):=K_{Y}+\Delta(f)$ : c'est un $\mathbb{Q}$-diviseur sur $Y$;

(2) $\kappa(Y / \Delta):=\kappa\left(Y, K_{Y}+\Delta\right) \geqslant \kappa(Y)$. 


\section{F. Campana et M. PăUn}

Si $f^{\prime}: X^{\prime} \rightarrow Y^{\prime}$ est une modification de $f$, on a $: \kappa\left(Y^{\prime} / \Delta\left(f^{\prime}\right)\right) \leqslant \kappa(Y / \Delta(f))$. On peut avoir inégalité stricte si $\kappa(Y)=-\infty$ (mais pas si $\kappa(Y) \geqslant 0$ ).

On dit que $f$ est une fibration de type général si $\kappa\left(Y^{\prime} / \Delta\left(f^{\prime}\right)\right)=\operatorname{dim}(Y)>0$ pour toute modification $f^{\prime}$ de $f$. Lorsque $\kappa(Y) \geqslant 0$ (et c'est le cas des exemples de Bogomolov-Tschinkel), il suffit de considérer $f$ seulement, la dimension canonique de la base orbifolde de $f^{\prime}$ ne dépendant alors pas du modèle biméromorphe $f^{\prime}$ de $f$ considéré. Voir [Cam04, Proposition 1.14, p. 514]. Cette notion est biméromorphe, et permet de définir $\kappa(f):=\kappa\left(Y^{\prime} / \Delta\left(f^{\prime}\right)\right)$ lorsque $f: X \rightarrow Y$ est maintenant une application méromorphe dominante à fibre générique connexe, entre variétés projectives irréductibes à singularités arbitraires, et $f^{\prime}: X^{\prime} \rightarrow Y^{\prime}$ un modèle biméromorphe de $f$ pour lequel la base orbifolde de $f^{\prime}$ est de dimension canonique minimum.

Definition 1.1. On dit que $X$ est spéciale s'il n'existe pas de fibration méromorphe de type général $f: X \rightarrow Y$

Les exemples fondamentaux de variétés spéciales sont les variétés rationnellement connexes et les variétés $X$ avec $\kappa(X)=0$. Inversement toute $X$ spéciale peut être canoniquement décomposée en tour de fibrations de l'un de ses deux types (dans un sens orbifolde adéquat, sous réserve de la validité de la conjecture $C_{n, m}^{\text {orb }}$ ).

On conjecture dans [Cam04, 9.2, 9.20] que les variétés spéciales sont les analogues de dimension supérieure des courbes rationnelles ou elliptiques :

(1) $X$ est spéciale si et seulement si sa pseudométrique de Kobayashi est nulle ;

(2) $X$ est spéciale si et seulement si elle est potentiellement dense (si définie sur un corps de nombres) ;

(3) si $X$ est spéciale, $\pi_{1}(X)$ est virtuellement abélien.

L'un des résultats principaux de [Cam04, 5.8, 5.16, pp. 579-582] est le théorème suivant.

ThÉorème 1.2. Soit $X$ projective, irréductible. Il existe une unique fibration $c_{X}: X \rightarrow C(X)$, appelée le coeur de $X$, telle que :

(1) les fibres générales de $X$ sont spéciales ;

(2) $c_{X}$ est soit une fibration de type général, soit constante (ceci si et seulement si $X$ est spéciale).

Le coeur scinde donc $X$ en ses deux composantes : spéciale (les fibres) et de type général (la base orbifolde). Il fournit une description synthétique de la géométrie de $X$ analogue à celle des surfaces due à Enriques-Shafarevitch-Kodaira.

Ce scindage a lieu aussi, conjecturalement, aux niveaux hyperbolique et arithmétique. On conjecture en effet dans [Cam04] en particulier qu' il existe un sous-ensemble algébrique strict $Z \subset C(X)$ tel que :

(1) si $h: \mathbb{C} \rightarrow X$ est une courbe entière de $X$, alors $c_{X} \circ h: \mathbb{C} \rightarrow C(X)$ est soit constante, soit d'image contenue dans $Z$;

(2) si $K$ est un corps de nombres sur lequel $X$ est définie (ainsi donc que $c_{X}$ ), alors l'intersection de $c_{X}(X(K))$ avec $U:=X-Z$ est fini.

Ces conjectures sont inspirées par celles de Lang pour les variétés de type général, et en sont la version orbifolde, par composition avec le coeur. 


\section{VARIÉTÉS FAIBLEMENT SPÉCIALES À COURBES ENTIÈRES DÉGÉNÉRÉES}

\section{La construction de Bogomolov-Tschinkel}

\subsection{Les exemples de Bogomolov-Tschinkel}

ThÉORÈme 2.1. Il existe une fibration elliptique $\varphi: X \rightarrow B$ dans laquelle $X, B$ sont projectives complexes lisses et connexes, $\operatorname{dim}(X)=3, \operatorname{dim}(B)=2$, telle que de plus :

(1) $\pi_{1}(X)=\{1\}$;

(2) $\kappa(B)=1$;

(3) $\kappa\left(B, K_{B}+\Delta(\varphi)\right)=2$.

Une telle fibration peut être définie sur $\mathbb{Q}$.

Une construction sera décrite dans la section suivante. On en déduit le corollaire suivant.

Corollaire 2.2. Pour $\varphi: X \rightarrow B$ comme ci-dessus : $X$ est $f$-spéciale, non spéciale, et $\varphi=c_{X}$ est le coeur de $X$.

Démonstration. La fibration $\varphi$ est à fibres génériques spéciales (courbes elliptiques), et base orbifolde de type général (par la propriété (3)). C'est donc le coeur de $X$, qui n'est donc pas spéciale. Pour montrer que $X$ est faiblement spéciale, il suffit, puisqu'elle est simplement connexe, de voir qu'elle n'a pas de fibration $f: X \rightarrow Y$ sur une base $Y$ de type général avec $d:=\operatorname{dim}(Y)>0$. On ne peut avoir ni $d=3$ (puisque $X$ est couverte par des courbes elliptiques), ni $d=1$ (puisque $X$ est simplement connexe). Si $d=2$, on a $f \neq \varphi$, puisque $\kappa(B)=1$; donc $Y$ est couverte par les images des fibres de $\varphi$, et ne peut donc être de type général. Contradiction.

L'objectif du présent texte est de montrer le théorème suivant.

ThÉORÈme 2.3. Il existe des fibrations $\varphi: X \rightarrow B$ possédant les propriétés énoncées dans le corollaire 2.2, telles qu'existe une courbe projective $\Gamma \subset B$ telle que pour toute courbe entière $h: \mathbb{C} \rightarrow X$, l'image de $\varphi \circ h: \mathbb{C} \rightarrow B$ est soit un point, soit contenue dans $\Gamma$ ('hyperbolicité transcendante').

En particulier, si $C \subset X$ est une courbe rationnelle ou elliptique tracée sur $X$, alors $\varphi(C) \subset B$ est soit un point, soit contenue dans $\Gamma$ ('hyperbolicité algébrique').

On peut trouver de tels $\varphi$ définies $\operatorname{sur} \mathbb{Q}$.

L'hyperbolicité algébrique sera établie dans la $\S 3$ par un argument très simple. La version transcendante le sera dans la $\S 4$.

\subsection{La construction}

Nous conservons les notations de [BT03]. Soit $\left(B, \varphi_{1}, \psi, \eta^{\prime}, E^{\prime}\right)$ un quintuplet tel que les conditions suivantes soient satisfaites.

BT1. $\eta^{\prime}: E^{\prime} \rightarrow C$ est une surface elliptique projective ayant une (unique) fibre multiple $\left(\eta^{\prime}\right)^{-1}(0):=$ $E_{0}^{\prime}$, de multiplicité $m \geqslant 2$, fixée dans la suite du texte. On suppose aussi que $E^{\prime}-E_{0}^{\prime}$ est simplement connexe. On peut obtenir de telles données par transformation logarithmique d'une surface rationnelle elliptique adéquate.

BT2. $B$ est une surface elliptique minimale projective $\varphi_{1}: B \rightarrow C_{1} \cong \mathbb{P}^{1}$ avec $\kappa(B)=1$, telle que, de plus :

BT3. Il existe une fibration $\psi: B \rightarrow \mathbb{P}_{1}$, distincte de $\varphi_{1}$, dont une fibre lisse $D:=\psi^{-1}(0)$ (nécessairement de genre $g \geqslant 2$ ) soit lisse et telle que $B-D$ soit simplement connexe. 


\section{F. Campana et M. PăUN}

La construction de [BT03] consiste alors à prendre pour $\varphi: X:=E^{\prime} \times_{\mathbb{P}_{1}} B \rightarrow B$ la fibration déduite de $\eta^{\prime}: E^{\prime} \rightarrow \mathbb{P}_{1}$ par le changement de base $\psi: B \rightarrow \mathbb{P}_{1}$, après normalisation du produit fibré.

On a donc : $\Delta(\varphi)=\psi^{-1}\left(\Delta\left(\eta^{\prime}\right)\right)=\psi^{-1}\left((1-1 / m) \cdot E_{0}^{\prime}\right)=(1-1 / m) \varphi^{-1}(D)$. On en déduit immédiatement les propriétés énoncées en corollaire 2.2 (la simple connexité de $X$ résulte de celle de $\left.X-\varphi^{-1}(D)\right)$.

Montrons maintenant, suivant [BT03], comment réaliser la simple-connexité de $B-D$. Dans la suite, nous considérerons toujours cette situation particulière.

BT4. Soit $B_{0}$ une surface projective lisse et simplement connexe telle que $\kappa\left(B_{0}\right)=1$. Soit $D_{0}, D_{0}^{\prime} \in$ $\left|L_{0}\right|$ deux membres génériques (et même éventuellement généraux), où $L_{0} \in \operatorname{Pic}\left(B_{0}\right)$ est ample, et $\left|L_{0}\right|$ soit sans point base.

Soit alors $\psi: B_{0} \rightarrow C \cong \mathbb{P}^{1}$ l'application méromorphe définie par le système linéaire de dimension 1 engendré par $D_{0}$ et $D_{0}^{\prime}$, qui se coupent transversalement en un ensemble fini noté $F$. On note $\beta: B \rightarrow B_{0}$ l'éclatement de $B_{0}$ le long de $F$, et $D \subset B$ le transformé strict de $D_{0}$.

Montrons la simple connexité de $(B-D)$. Par le théorème de Lefschetz, $\pi_{1}\left(B_{0}-D_{0}\right) \cong \mathbb{Z}_{r}$, si $r>0$ est le plus grand entier par lequel $L_{0}$ est divisible dans $\operatorname{Pic}\left(B_{0}\right)$. Et $\pi_{1}\left(B_{0}-D_{0}\right)$ est engendré par un lacet $g$ tournant une fois autour de $D_{0}$. Le relevé par $\beta$ de ce lacet engendre donc $\pi_{1}(B-D)$, mais devient homotope à zéro dans $P-\{u\} \cong \mathbb{C}$, si $P$ est une courbe exceptionnelle de $\beta$, et $u$ son point d'intersection avec $D$. D'où la simple connexité.

On fixera toujours $S_{0}, L_{0}, D_{0}$ dans la suite. Par contre, $D_{0}^{\prime}$ sera variable et devra être choisi générique (ou général) dans le système linéaire défini par $L_{0}$. On notera donc quelquefois $B_{F}$ la surface $B$, et $\varphi_{F}: X_{F} \rightarrow B_{F}$ la fibration associée pour indiquer la dépendance en $F$.

Remarque 2.4. Dans [BT03], les auteurs imposent au pinceau défini par $D_{0}, D_{0}^{\prime}$ d'être très ample, et d'avoir tous ses membres irréductibles. La seconde condition n'est pas nécessaire. La première peut être affaiblie en : $D_{0}, D_{0}^{\prime}$ sont amples et membres génériques d'un système linéaire sans point base (comme ci-dessus). La seule propriété utilisée est en effet que le groupe fondamental du complémentaire de $D_{0}$ dans $S$ soit fini et engendré par un petit lacet tournant une fois autour de $D_{0}$, également satisfaite sous cette hypothèse plus faible.

Dans la suite, nous aurons besoin de propriétés additionnelles BT5 et BT6, que nous introduisons maintenant.

BT5. On supposera toujours que $L_{0}$ est divisible par $m \geqslant 2$ (la multiplicité de $E_{0}^{\prime}$ dans $\eta^{\prime}$ ). Il existe donc un revêtement cyclique $r: B^{\prime} \rightarrow B_{0}$, de degré $m$, ramifié au-dessus de $D_{0}$ exactement. Puisque $D_{0}$ est lisse, $B^{\prime}$ l'est aussi.

BT6. On supposera aussi que $\left(c_{1}^{2}-c_{2}\right)\left(B^{\prime}\right)>0$.

Montrons comment réaliser BT6 aussi.

\subsection{Exemples}

Nous appliquerons deux fois le lemme suivant :

Lemme 2.5. Soit $S$ une surface complexe lisse, compacte et connexe. Soit $D \in|m L|$ un diviseur lisse, et $r: S^{\prime} \rightarrow S$ le revêtement cyclique de degré $m$ de $S$ ramifié le long de $D$. Les nombres de Chern $c_{1}^{2}\left(S^{\prime}\right)$ et $c_{2}\left(S^{\prime}\right)$ de $S^{\prime}$ satisfont alors les relations suivantes (ceux de $S$ étant notés $c_{1}^{2}$ et $c_{2}$ ) :

(1) $c_{1}^{2}\left(S^{\prime}\right)=m \cdot\left[c_{1}^{2}+(m-1) \cdot\left(2 \cdot K_{S} \cdot L+(m-1) \cdot L^{2}\right)\right]$;

(2) $c_{2}\left(S^{\prime}\right)=m \cdot\left[c_{2}+(m-1) \cdot\left(K_{S} \cdot L+m \cdot L^{2}\right)\right]$;

(3) $c_{1}^{2}\left(S^{\prime}\right)-c_{2}\left(S^{\prime}\right)=m \cdot\left[\left(c_{1}^{2}-c_{2}\right)+(m-1)\left(K_{S} \cdot L-L^{2}\right)\right]$. 


\section{VARIÉTÉS FAIBLEMENT SPÉCIALES À COURBES ENTIÈRES DÉGÉNÉRÉES}

Démonstration. On a, par formule de ramification :

$$
K_{S^{\prime}}=r^{*}\left(K_{S}+(m-1) \cdot L\right), \quad \operatorname{donc} c_{1}^{2}\left(S^{\prime}\right)=m \cdot\left[c_{1}^{2}+2(m-1) \cdot K_{S} \cdot L+(m-1)^{2} L^{2}\right] .
$$

Pour calculer $c_{2}\left(S^{\prime}\right)$, on remarque que $\chi_{t}=c_{2}$ étant la caractéristique topologique, la suite exacte de Mayer-Vietoris fournit $: c_{2}\left(S^{\prime}\right)=\chi_{t}\left(S^{\prime}\right)=m \cdot \chi_{t}(S)-(m-1) \cdot \chi_{t}(D)=m \cdot c_{2}(S)+$ $(m-1) \cdot\left[K_{S} \cdot D+D^{2}\right]=m \cdot c_{2}(S)+(m-1) \cdot m \cdot\left[K_{S} \cdot L+m \cdot L^{2}\right]$.

Corollaire 2.6. Dans la situation du lemme précédent, $c_{1}^{2}\left(S^{\prime}\right)-c_{2}\left(S^{\prime}\right)>0$ si les conditions suivantes $P 1$ et $P 2$ sont satisfaites :

P1. $K_{S} \cdot L>L^{2}$;

P2. $m>\left(1-\left[\left(c_{1}^{2}-c_{2}\right) /\left(K_{S} \cdot L-L^{2}\right)\right]\right)$.

Nous allons montrer sur des exemples très simples que les conditions du corollaire précédent peuvent être réalisées avec des surfaces elliptiques minimales dans la situation de corollaire 2.2.

Soit $s: B_{0} \rightarrow P:=\mathbb{P}^{1} \times \mathbb{P}^{1}$ le revêtement double ramifié le long du diviseur lisse $R \in$ $\left|\mathcal{O}_{P}(2(k+2), 4)\right|$. On suppose $R$ (et donc $S$ ) lisse. Par le lemme 1.1, on a donc : $c_{1}^{2}\left(B_{0}\right)=0$, et $c_{2}\left(B_{0}\right)=12 \chi\left(\mathcal{O}_{S}\right)=12 .(k+2)$.

On vérifie de plus que $B_{0}$ est simplement connexe, par [FM94, II, 2.2, Proposition 2.1].

On choisit ensuite $L_{0}:=s^{*}\left(L^{\prime}\right)=s^{*}\left(O_{P}(b, b a)\right)$, pour $a, b>0$ entiers. On a donc : $K_{B_{0}} \cdot L_{0}=$ $2 k b a$, et $L_{0}^{2}=4 b^{2} a$, de sorte que la propriété P1 (respectivement P2) précédente est satisfaite si $k \geqslant(2 b+1)$, et si $(m-1) \cdot a>12 \cdot((k+2) /(k-2 b))$.

\section{Hyperbolicité algébrique}

Hypothèses 3.0. On fixe $\varphi: X \rightarrow B$, construite comme dans le corollaire 2.2. On suppose que les conditions BT1-BT6 sont satisfaites. En particulier : $\left(c_{1}^{2}-c_{2}\right)\left(B^{\prime}\right)>0$.

On appelle courbe rationnelle (respectivement elliptique) une courbe projective irreductible complexe $C$ de genre géométrique $g(\hat{C}):=0$ (respectivement 1), si $\hat{C}$ est la normalisée de $C$.

On note $R \subset B_{0}$ la réunion (dénombrable) des courbes rationnelles ou elliptiques de $B_{0}$. Donc $R \cap D_{0}$ est un ensemble dénombrable puisque $g\left(D_{0}\right)>1$. Puisque $L_{0}$ est, par hypothèse, sans point base, lorsque $D_{0}^{\prime}$ est général dans $\left|L_{0}\right|, F:=D_{0}^{\prime} \cap D_{0}$ est disjoint de $R \cap D_{0}$.

ThÉorème 3.1. On suppose les hypothèses 3.0 ci-dessus réalisées, et $D_{0}^{\prime}$ disjoint de $\left(D_{0} \cap R\right)$. Il existe une courbe projective $Z \subset B$ telle que, pour toute courbe rationnelle ou elliptique $C$ de $X$, $\varphi(C) \subset B$ est soit un point, soit contenue dans $Z$.

Démonstration. Soit $\varphi^{\prime}: X^{\prime}:=X \times{ }_{B} B^{\prime} \rightarrow B^{\prime}$ la (normalisée de la) fibration déduite de $\gamma:=\beta \circ \varphi$ : $X \rightarrow B_{0}$ par le changement de base $r: B^{\prime} \rightarrow B_{0}$ défini en $\S 2.2$, propriété BT5. Soit $u: X^{\prime} \rightarrow X$ la projection naturelle. Alors $u$ est étale au-dessus de $\left(B_{0}-F\right)$, si $F \subset B_{0}$ est l'ensemble fini intersection de $D_{0}$ et $D_{0}^{\prime}$.

Par le choix de $D_{0}^{\prime}, \varphi(C)$ ne rencontre pas $F$, si $C$ n'est pas contenue dans une fibre de $\varphi$ (ce que nous supposerons désormais).

Donc, $u$ est étale au-dessus de $C$, et $C^{\prime}:=u^{-1}(C)$ est une réunion (finie) de courbes rationnelles ou elliptiques de $X^{\prime}$. Il en est de même de l'image $C^{\prime \prime}:=\varphi^{\prime}\left(C^{\prime}\right) \subset B^{\prime}$ de $C^{\prime}$ par $\varphi^{\prime}: X^{\prime} \rightarrow B^{\prime}$. De plus, aucune composante irréductible de $C^{\prime \prime}$ n'est un point de $B^{\prime}$, puisque $C$ n'est pas contenue dans une fibre de $\varphi$.

Or, $c_{1}^{2}\left(B^{\prime}\right)-c_{2}\left(B^{\prime}\right)>0$, d'après théorème 2.5. On déduit de [Bog77] (voir aussi [Des77]) qu'il existe une courbe $Z^{\prime} \subset B^{\prime}$, réunion finie de courbes rationnelles ou elliptiques, qui contient $C^{\prime \prime}$. Donc $\varphi(C) \subset Z$, si $Z:=\beta^{-1}\left(r\left(Z^{\prime}\right)\right)$. 


\section{F. CAmpana Et M. PĂUN}

\section{Hyperbolicité, version transcendante}

\subsection{Notion de $m$-tangence}

Soit $S_{0}, L_{0}, F, B, B^{\prime}, D, X, \varphi$ définis comme en $\S 2.2$. On suppose les propriétés BT1-BT6 satisfaites.

Definition 4.1. Soit $h: M \rightarrow X$ une application holomorphe d'une courbe complexe (variété analytique complexe lisse et connexe de dimension pure 1) dans $B$. On dit que $h$ (ou $M$, par abus de language) est (classiquement) $m$-tangente à $D$ si l'ordre de contact de $h(M)$ et de $D$ en chacun de leurs points d'intersection est divisible par $m$ (ceci après composition avec la normalisation de $M$ ). Plus précisément : si $h^{*}(D)$ est un diviseur effectif de $M$ dont les coefficients sont tous divisibles par $m$. Si $C$ est une courbe complexe (éventuellement singulière) tracée sur $X, h$ sera la composée de l'inclusion naturelle de $C$ dans $X$ avec une normalisation de $C$.

Remarque. La propriété de $m$-tangence non classique est définie de manière similaire, mais en éxigeant seulement un ordre de contact au moins égal à $m$. Bien que plus naturelle, elle n'est pas utilisée ici. Voir [Cam05] pour les différences (parfois essentielles) entre les deux notions.

L'observation triviale suivante permet de réduire à l'orbifolde $(B / \Delta(\varphi))$ l'étude des courbes tracées sur $X$.

ThÉorème 4.2. On suppose les hypothèses 3.0 ci-dessus réalisées. Soit $h: M \rightarrow X$ une application holomorphe de la courbe complexe $M$ vers $X$, telle que $\varphi \circ h: M \rightarrow B$ soit non constante. Alors : $\varphi \circ h: M \rightarrow B$ est (classiquement) $m$-tangente à $D$.

Démonstration. Soit $H:=\varphi^{-1}(D)$ l'image réciproque réduite de $D$. C'est un diviseur irréductible, et $\varphi^{*}(D)=m . H$, puisque les fibres de $\varphi$ au-dessus des points de $D$ sont toutes irréductibles de multiplicité $m$. On a donc : $(\varphi \circ h)^{*}(D)=h^{*}\left(\varphi^{*}(D)\right)=h^{*}(m \cdot H)=m \cdot h^{*}(H)$, et l'assertion.

\subsection{Objectif}

ThÉorème 4.3. Soit $S_{0}, L_{0}, D_{0}, B^{\prime}$ possédant les propriétés BT1-BT6 énoncées au $\S 2.2$. Pour $D_{0}^{\prime}$ générique dans $\left|L_{0}\right|$, soit $\varphi_{F}: X_{F} \rightarrow B_{F}$ (voir notations BT5 de la $\S 2.2$ ) la fibration associée.

Alors il existe une courbe algébrique $\Gamma \subset B=B_{F}$ telle que pour toute courbe entière $h: \mathbb{C} \rightarrow B$ $m$-tangente à $D$ est soit constante, soit d'image contenue dans $\Gamma$.

En particulier, si $h: \mathbb{C} \rightarrow X$ est une courbe entière, alors $\varphi \circ h: \mathbb{C} \rightarrow B$ est soit constante, soit d'image contenue dans $\Gamma$.

Nous allons pour ceci adapter au cadre orbifolde des arguments de théorie de Nevanlinna. Le point clé (tout comme dans la section précédente) est que l'orbifolde $(B / \Delta)$, de dimension 2 , avec $\Delta:=(1-1 / m) . D$ est de type général avec $\left(c_{1}^{2}-c_{2}\right)>0$ (dans un sens orbifolde adéquat).

Nous ne définirons ici que les notions de formes différentielles orbifolde effectivement utilisées (bien qu'il soit possible de travailler de façon similaire dans un contexte général).

Expliquons pourquoi il n'est pas possible ici d'appliquer au revêtement cyclique ramifié de $S$ le long de $D$ les arguments très simples de la section précédente pour obtenir la version transcendante de l'hyperbolicité : c'est que les courbes entières de $B$ (en général non algébriquement dégénérées) peuvent recouvrir $B$, et que l'on ne peut alors pas choisir $F=D \cap D^{\prime}$ disjoint de toutes ces courbes entières. Et l'on ne peut alors pas nécessairement relever à $X^{\prime}$ (déduit par changement de base par ce revêtement) les courbes entières de $X$.

On montre néammoins l'éxistence d'un nombre fini de points de $D$ tels que toute courbe entière tracée sur $B$ et $m$-tangente à $D$ coupe $D$ en ces points seulement au plus. Cette étape repose sur la théorie de Nevanlinna, appliquée aux formes pluridifférentielles orbifolde sur $(B / \Delta)$. On utilise, suivant l'argument original de Bogomolov, l'inégalité $\left(c_{1}^{2}-c_{2}\right)>0$ (dans un sens orbifolde adéquat) 


\section{VARIÉTÉS FAIBLEMENT SPÉCIALES À COURBES ENTIÈRES DÉGÉNÉRÉES}

pour produire des feuilletages sur $B$ auquel toute courbe entière (ou bien rationnelle ou elliptique) doit être tangente.

\subsection{Formes à pôles logarithmiques orbifoldes}

On se donne une surface projective complexe lisse et connexe $B$, une courbe lisse et irréductible $D$ sur $B$, et une multiplicité entière $m \geqslant 2$. Le diviseur orbifolde considéré sur $B$ est $\Delta:=(1-1 / m)$. $D$.

Notre objectif est le Corollaire 4.4 ci-dessous, qui fournit des conditions suffisantes pour l'existence de formes pluridifférentielles sur l'orbifolde $(B / \Delta)$.

Corollaire 4.4. Soit $B, D, m$ comme ci-dessus, et $A$ 'big' sur $B$. On suppose que $\kappa(B)=1$, que $g(D)>1$, que $\mathcal{O}_{D}(D) \cong \mathcal{O}_{D}$, et que $\left(c_{1}^{2}-c_{2}\right)(B)+(1-1 / m) \cdot K_{B} \cdot D>0$.

Alors, il existe $N>0$ tel que $H^{0}\left(B,\left[\operatorname{Sym}^{N}\right]\left(\Omega_{(B / \Delta)}^{1}\right) \otimes A^{-1}\right) \neq 0$.

Remarquons que les hypothèses du précédent corollaire sont satisfaites lorsque les données $B, D, m$ sont déduites par éclatement de $F$ de données $B_{0}, L_{0}, D_{0}, F$ satisfaisant les hypothèses BT1-BT6 de la $\S 2.2$.

Expliquons brièvement l'origine de la notion de forme pluridifférentielle sur l'orbifolde $(B / \Delta)$ (sous la forme implicite rudimentaire utilisée ici).

Si $c_{1}(D)$ est divisible par $m$ dans $N S(B)$, on peut considérer le revêtement cyclique $r: B^{\prime} \rightarrow B$ de degré $m$ ramifié le long de $D$.

Si $(x, y)$ sont des coordonnées locales holomorphes près d'un point $a \in D$, en lequel $D$ a pour équation $y=0$, et si $a^{\prime}:=r^{-1}(a) \in S^{\prime}$, alors les sections locales de $\operatorname{Sym}^{N}\left(\Omega_{B^{\prime}}^{1}\right)$ s'écrivent sous la forme

$$
\sum_{j=0}^{j=N} a_{j} \cdot\left(d\left(y^{1 / m}\right)\right)^{\otimes j} \otimes d x^{\otimes(N-j)}=\sum_{j=0}^{j=N} a_{j} \cdot\left(d y / y^{(1-1 / m)}\right)^{\otimes j} \otimes d x^{\otimes(N-j)},
$$

où $y^{1 / m}$ est une coordonnée locale de $B^{\prime}$ en $a^{\prime}$ telle que $\left(y^{1 / m}\right)^{m}=r^{*}(y)$.

Ceci suggère de prendre la partie entière de cette expression, c'est-à-dire la somme des termes précédents, mais en y remplaçant les exposants fractionnaires $(1-1 / m) \cdot j$ par leur partie entière $[j-j / m]$. On obtient ainsi par image réciproque par $r$ des sections de $\operatorname{Sym}^{N}\left(\Omega_{B^{\prime}}^{1}\right)$.

Definition 4.5. Soit $\left[\operatorname{Sym}^{N}\right]\left(\Omega_{(B / \Delta)}^{1}\right)$ le faisceau des germes de sections méromorphes de $\operatorname{Sym}^{N}\left(\Omega_{B}^{1}\right)$ qui sont holomorphes en dehors de $D$, et qui s'écrivent en coordonnées locales $(x, y)$ près de $a \in D$ sous la forme :

$$
\sum_{j=0}^{j=N} a_{j} \cdot\left(d y^{\otimes j} / y^{[(1-1 / m) j]}\right) \otimes d x^{\otimes(N-j)}=\sum_{j=0}^{j=N} a_{j} \cdot y^{[j / m]+\epsilon(j, m)} \cdot(d y / y)^{\otimes j} \otimes d x^{\otimes(N-j)},
$$

avec les $a_{j}$ holomorphes, où [*] est la partie entière, et où $\epsilon(j, m):=0$ (respectivement 1 ) si $m$ divise $j$ (respectivement : sinon).

Notons que $[j / m]+\epsilon(j, m)$ vaut 0 si $j=0$; vaut 1 si $1 \leqslant j \leqslant m$; et vaut $k$ si et seulement si $(k-1) m+1 \leqslant j \leqslant k m$.

Afin de faciliter l'écriture, on pose en $4.2-4.5$ ci-dessous : $\left[\operatorname{Sym}^{N}\right]\left(\Omega_{(B / \Delta)}^{1}\right):=S^{N}\left(m, B_{F}\right)=$ $S^{N}(m)$, donc $S^{N}(\infty):=\operatorname{Sym}^{N}\left(\Omega_{B}^{1}(\log D)\right)$.

Remarque 4.6. Observons que :

- L'expression ci-dessus conserve la même forme si l'on change les coordonnées locales $(x, y)$, avec $y=0$ équation locale de $D$. 


\section{F. Campana et M. PăUN}

- Rappelons que $\Omega_{B}^{1}(\log D)$ est le faisceau précédent, lorsque $m=\infty$, et que $\left[\operatorname{Sym}^{N}\right]\left(\Omega_{(B / \Delta)}^{1}\right) \subset$ $\operatorname{Sym}^{N}\left(\Omega_{B}^{1}(\log D)\right)$.

Plus généralement, si $\Delta=(1-1 / m)$. $D$ et si $\Delta^{\prime}=\left(1-1 / m^{\prime}\right)$. $D$, pour $1 \leqslant m \leqslant m^{\prime} \leqslant \infty$, alors $\left[\operatorname{Sym}^{N}\right]\left(\Omega_{(B / \Delta)}^{1}\right) \subset\left[\operatorname{Sym}^{N}\right]\left(\Omega_{\left(B / \Delta^{\prime}\right)}^{1}\right)$.

- L'application naturelle de multiplication : $S^{N}(m) \otimes S^{M}(m) \rightarrow S^{N+M}(m)$, qui envoit

$$
\left[\left(\sum_{j=0}^{j=N} a_{j} \cdot\left(d y^{\otimes j} / y^{[(1-1 / m) j]}\right) \otimes d x^{\otimes(N-j)}\right)\right] \otimes\left[\sum_{k=0}^{k=M} b_{k} \cdot\left(d y^{\otimes k} / y^{[(1-1 / m) k]}\right) \otimes d x^{\otimes(M-j)}\right]
$$

sur :

avec

$$
\left[\sum_{j=0}^{j=N+M} c_{h} \cdot\left(d y^{\otimes h} / y^{[(1-1 / m) h]}\right) \otimes d x^{\otimes(N+M-h)}\right]
$$

est bien définie, puisque :

$r(j, k ; m):=[j / m]+\epsilon(j, m)+[k / m]+\epsilon(k, m)-\{[(j+k) / m]+\epsilon(j+k, m)\} \geqslant 0$, pour tous $j, k, m$, par un calcul immédiat.

Proposition 4.7. Si $\mathcal{O}_{D}(D) \cong \mathcal{O}_{D}$, et si g est le genre de $D$, alors :

$$
h^{0}\left(B, S^{q m}(m)\right) \geqslant h^{0}\left(B, S^{q m}(\infty)\right)-\left[\left((2(g-1)) \cdot m^{2} q^{3}\right) / 6+A(m) q^{2}\right],
$$

où $A(m)$ est une constante ne dépendant que de $m$.

Démonstration. Le faisceau quotient $Q:=S^{N}(\infty) / S^{N}(m)$ est supporté par $D$, et $h^{0}\left(B, S^{q m}(m)\right) \geqslant$ $h^{0}\left(B, S^{q m}(\infty)\right)-h^{0}(D, Q)$.

Nous allons montrer que $h^{0}(D, Q) \leqslant\left(\left((2(g-1)) \cdot m^{2} q^{3}\right) / 6-A(m) q^{2}\right.$, ce qui établira l'assertion.

Le faisceau $Q$ admet une filtration naturelle $Q_{N} \subset Q_{N-1} \subset \cdots \subset Q_{1} \subset Q_{0}=Q$ telle que, pour $j=0,1, \ldots, N$, on ait : $Q_{(j-1)} / Q_{j}=\operatorname{Sym}^{N-j}\left(\Omega_{D}^{1}\right) \otimes F_{j}$, où $F_{j}$ est un faisceau localement libre de rang $r_{j}:=[j / m]+\epsilon(j, m)$, et admettant une filtration $F_{j, 0} \subset F_{j, 1} \subset \cdots \subset F_{j, r_{j}}=F_{j}$, avec $F_{j, h} / F_{j, h-1} \equiv\left(N^{*}\right)^{\otimes h}$, où $N^{*}$ est le dual du fibré normal à $D$ dans $B$.

Nous n'utiliserons ces filtrations que lorsque $N \cong \mathcal{O}_{D}$.

Dans ce cas, on a donc :

$$
h^{0}\left(D, Q_{j}\right) \leqslant r_{j} \cdot h^{0}\left(D, K_{D}^{\otimes(N-j)}\right) \leqslant(2(N-j)-1)(g-1) . r_{j}, \quad \text { si } N-j \geqslant 2 .
$$

On suppose désormais que $N=q m$ est un multiple de $m$.

On a alors (puisque $1 \leqslant h:=r_{j} \leqslant q$ si $j=(h-1) \cdot m+k$, avec $1 \leqslant k \leqslant m$ ) :

$$
\begin{aligned}
h^{0}(D, Q) & \leqslant \sum_{j=1}^{j=N} h^{0}\left(D, Q_{j}\right)=1+\sum_{h=1}^{h=q} h \cdot\left[\sum_{k=1}^{k=m} h^{0}\left(D, K_{D}^{\otimes((q-h+1) m-k)}\right)\right] \\
& \leqslant \sum_{h=1}^{h=q} m h \cdot(2(q-h+1) m-1)(g-1) .
\end{aligned}
$$

Cette dernière somme vaut : $\left(2(g-1) m^{2} \cdot q^{3}\right)((1 / 2)-(1 / 3))-A(m) q^{2}$, où $A(m)$ dépend de $m$ seulement, ce qui établit l'assertion.

Proposition 4.8. Soit $B, D, m$ comme ci-dessus, et $F:=\Omega_{B}^{1}(\log D)$.

On pose $: e_{1}:=c_{1}(F)$ et $e_{2}:=c_{2}(F)$, ainsi que $c_{1}:=c_{1}(B)$ et $c_{2}:=c_{2}(B)$. 


\section{VARIÉTÉS FAIBLEMENT SPÉCIALES À COURBES ENTIÈRES DÉGÉNÉRÉES}

On suppose que $\mathcal{O}_{D}(D) \cong \mathcal{O}_{D}$. (Donc $2(g-1)=K_{B} . D$. $)$

Alors :

(1) $e_{1} \equiv-c_{1}+D, e_{1}^{2}=c_{1}^{2}+2 K_{B} \cdot D$;

(2) $e_{2}=c_{2}+K_{B} \cdot D, e_{1}^{2}-e_{2}=c_{1}^{2}-c_{2}+K_{B} \cdot D$;

(3) $h^{0}\left(B, S^{q m}(m)\right) \geqslant \alpha \cdot(m q)^{3} / 6-O\left(q^{2}\right)$, si $\alpha:=\left(c_{1}^{2}-c_{2}\right)+(1-1 / m) \cdot K_{B} \cdot D>0$, et si $\kappa(B)=1$ et $g(D) \geqslant 2$.

Démonstration. Le faisceau quotient $R:=\Omega_{B}^{1}(\log D) / \Omega_{B}^{1}$ est supporté sur $D$ et isomorphe à $\mathcal{O}_{D}$. Donc $e_{1}=-c_{1}+D$ (restreindre à une courbe très ample arbitraire).

Pour calculer $e_{2}$, on applique la formule de Riemann-Roch à l'égalité $\chi\left(\Omega_{B}^{1}(\log D)\right)=\chi\left(\Omega_{B}^{1}\right)+$ $\chi\left(\mathcal{O}_{D}\right)$, en observant que $K_{B} \cdot D=-2 \chi\left(\mathcal{O}_{D}\right)$.

On obtient ainsi la seconde assertion, puisque cette égalité s'écrit :

$$
\left(e_{1}^{2} / 2\right)-e_{2}-K_{B} \cdot e_{1} / 2=\left(\left(c_{1}^{2} / 2\right)-c_{2}+K_{B} \cdot c_{1} / 2\right)-K_{B} \cdot D / 2,
$$

que $e_{1}^{2} / 2=c_{1}^{2} / 2+K_{B} \cdot D$, et que $-K_{B} \cdot e_{1} / 2=K_{B} \cdot c_{1} / 2-K_{B} \cdot D / 2$.

La dernière assertion résulte des deux propositions précédentes, de l'égalité de Riemann-Roch : $\chi\left(B, \operatorname{Sym}^{N}\left(\Omega_{B}^{1}(\log D)\right)\right)=\left(\left(e_{1}^{2}-e_{2}\right) / 6\right) \cdot N^{3}+O\left(N^{2}\right)$, et du lemme ci-dessous, en remarquant que :

$$
h^{0}\left(B,\left(\operatorname{Sym}^{M}\left(\Omega_{B}^{1}(\log D)\right)\right)\right) \geqslant \chi\left(B,\left(\operatorname{Sym}^{M}\left(\Omega_{B}^{1}(\log D)\right)\right)\right)-(1+g(D)),
$$

puisque :

$$
h^{2}\left(B,\left(\operatorname{Sym}^{M}\left(\Omega_{B}^{1}(\log D)\right)\right)\right) \leqslant 1+g(D) .
$$

Lemme 4.9. Soit $B$ une surface projective complexe lisse et connexe, et $D \subset B$ une courbe projective lisse et connexe de $B$. On suppose que $g(D) \geqslant 2$, et que $N:=\mathcal{O}_{D}(D) \cong \mathcal{O}_{D}$. Soit $T_{B}(-\log D)$ (respectivement $T_{B}(-D)$ ) le faisceau des germes de champs de vecteurs holomorphes sur $B$ qui sont tangents à $D$ (respectivement nuls le long de $D$ ).

Alors, pour tout $M>0$ :

(1) l'injection naturelle suivante a un conoyau de dimension $1+g(D)$ au plus :

$$
H^{0}\left(B,\left(\operatorname{Sym}^{M}\left(T_{B}(-D)\right)\right) \otimes K_{B}\right) \rightarrow H^{0}\left(B,\left(\operatorname{Sym}^{M}\left(T_{B}(-\log D)\right)\right) \otimes K_{B}\right) ;
$$

(2) si $B$ est une surface elliptique, alors :

$$
h^{2}\left(B,\left(\operatorname{Sym}^{M}\left(\Omega_{B}^{1}(\log D)\right)\right)\right)=h^{0}\left(B,\left(\operatorname{Sym}^{M}\left(T_{B}(-\log D)\right)\right) \otimes K_{B}\right) \leqslant 1+g(D) .
$$

Démonstration. Soit $T:=T_{B}(-\log D)$, et $T_{\mid D}$ sa restriction à $D$ (comme faisceau localement libre de rang 2).

On a deux suites exactes naturelles:

$$
0 \rightarrow T \rightarrow T_{B} \rightarrow N \rightarrow 0,
$$

et par restriction à $D$ :

$$
0 \rightarrow T_{D} \rightarrow T_{\mid D} \rightarrow N(-D) \cong \mathcal{O}_{D} \rightarrow 0 .
$$

Donc $\operatorname{Sym}^{M}\left(T_{\mid D}\right) \otimes K_{B}$ admet une filtration croissante par les $F_{j}, j=0,1, \ldots, M$ telle que $F_{j} / F_{j-1} \cong\left(T_{D}\right)^{\otimes j} \otimes K_{D}$, de sorte que $H^{0}\left(D, F_{j} / F_{j-1}\right)=0$ pour $j \leqslant M-2$.

Et que $H^{0}\left(D, F_{j} / F_{j-1}\right)=1$ (respectivement $\left.g(D)\right)$ si $j=M-1$ (respectivement $j=M$ ).

Donc : $h^{0}\left(D, \operatorname{Sym}^{M}\left(T_{\mid D}\right) \otimes K_{D}\right) \leqslant \sum_{0 \leqslant j \leqslant M} h^{0}\left(D, F_{j} / F_{j-1}\right)=1+g(D)$. 


\section{F. Campana et M. PăUN}

D'où la première assertion, en considérant la suite exacte :

$$
\begin{aligned}
H^{0}\left(B,\left(\operatorname{Sym}^{M}\left(T_{B}(-D)\right)\right) \otimes K_{B}\right) & \rightarrow H^{0}\left(B,\left(\operatorname{Sym}^{M}\left(T_{B}(-\log D)\right)\right) \otimes K_{B}\right) \\
& \rightarrow H^{0}\left(D,\left(\operatorname{Sym}^{M}\left(T_{B}(-\log D)_{\mid D}\right)\right) \otimes K_{D}\right) .
\end{aligned}
$$

Pour la seconde assertion, on note $g: B \rightarrow G$ la fibration elliptique de $B$. Alors $g: D \rightarrow G$ est finie, et $H^{0}\left(F,\left(\operatorname{Sym}^{M}\left(T_{B}(-\log D)_{\mid F}\right)\right) \otimes K_{B \mid F}\right)=0$, si $F$ est une fibre générique (lisse elliptique) de $g$, puisque $K_{B \mid F} \cong \mathcal{O}_{F}$.

Donc $g_{*}\left(\operatorname{Sym}^{M}\left(T_{B}(-D)\right) \otimes K_{B}\right)=0=H^{0}\left(B,\left(\operatorname{Sym}^{M}\left(T_{B}(-D)\right)\right) \otimes K_{B}\right)$, et enfin : $h^{0}\left(B,\left(\operatorname{Sym}^{M}\left(T_{B}(-\log D)\right)\right) \otimes K_{B}\right) \leqslant h^{0}\left(B,\left(\operatorname{Sym}^{M}\left(T_{B}(-D)\right)\right) \otimes K_{B}\right)+(1+g(D)) \leqslant 1+g(D)$.

Le corollaire 4.4 résulte immédiatement des assertions précédentes.

\subsection{Un théorème de confinement pour les courbes holomorphes $m$-tangentes à $D$}

Soit $B_{0}, D_{0}$ une surface munie d'une courbe $D_{0}$, possédant les propriétés énoncées dans le $\S 2.2$. On éclate $B_{0}$ en $F=D_{0} \cap D_{0}^{\prime}$, pour un choix générique de $D_{0}^{\prime}$, obtenant $B, D$.

Soit $h: \mathbb{C} \rightarrow B$ une courbe entière $m$-tangente à $D$, ou encore qui définit un morphisme orbifolde vers $(B, \Delta)$, c'est-à-dire telle que

$$
h^{*} D=\sum_{j} \nu_{j} \delta_{z_{j}}
$$

où toutes les multiplicités $\nu_{j}$ sont divisibles par $m$. (Voir cependant la lemme 4.10).

Nous allons montrer que (pour un choix générique de $D_{0}^{\prime}$ ), les points d'intersection de $h(\mathbb{C})$ avec $D$ se projettent en un ensemble fini $G:=G(F)$ de $D_{0}$, indépendant, non seulement de $h$, mais aussi de $F$. Ces faits établis, on peut conclure exactement comme dans le cas de la version algébrique de l'hyperbolicité. Nous ne reviendrons donc pas sur cet argument.

La finitude et l'indépendance en $h$ seront démontrées dans un première étape. L'indépendance en $F$ sera établie dans une seconde étape, qui nécessite de raffiner les arguments de la première étape, et fait appel à des propriétés plus fines des courants, rappelées pour la commodité du lecteur.

\section{Première étape : construction de $G(F)$}

Considérons également un fibré ample $A$ sur la surface $B$, muni d'une métrique $\psi$ à courbure positive. D'après le corollaire 4.4, il existe une section $\omega \in H^{0}\left(B,\left[\operatorname{Sym}^{N}\right]\left(\Omega_{(B / \Delta)}^{1}\right) \otimes A^{-1}\right)$, non-nulle.

On utilise également le fibré $A$ pour définir la fonction caractéristique $r \rightarrow T_{A}(h, r)$ de la courbe $h$ comme suit (voir [GK73] pour une présentation plus détaillée de cette notion)

$$
T_{A}(h, r):=\int_{0}^{r} \frac{d t}{t} \int_{D(t)} h^{*} \Theta_{\psi}(A)
$$

pour chaque $r \in \mathbb{R}_{+}$.

L'hypothèse sur le diviseur $h^{*} D$ ci-dessus montre qu'on peut faire agir $\omega$ sur la dérivée de la courbe (ou application) $h$, obtenant une section holomorphe de $h^{*} A^{-1}$. Le lemme suivant montre que $\omega$ fournit une équation différentielle algébrique pour la courbe $h$.

Lemme 4.10. La section $\omega\left(h^{\prime}\right)$ du fibré $h^{*} A^{-1}$ s'annule identiquement sur $\mathbb{C}$.

Démonstration. La démonstration répose sur la proposition suivante, due à Wong et Siu (voir [Won99, Ru01, Siu97]). On remarquera que les arguments classiques de courbure négative ne fonctionnent dans ce cadre qu'en utilisant une version orbifolde adéquate de la notion de métrique 


\section{VARIÉTÉS FAIBLEMENT SPÉCIALES À COURBES ENTIÈRES DÉGÉNÉRÉES}

hermitienne sur un fibré en droites (approche que nous n'avons pas développée ici), ceci à cause des pôles de $\omega$.

Proposition 4.11. Soit $\omega$ une différentielle méromorphe sur une variété projective $B$, qui admet des pôles logarithmiques le long d'un diviseur à croisements normaux $D$. Soit $h: \mathbb{C} \rightarrow B$ une courbe entière d'image non contenue dans $D$. Alors on a :

$$
\int_{0}^{2 \pi} \log ^{+}\left|\omega\left(h^{\prime}(r \exp (i \theta))\right)\right|_{\psi^{-1}} d \theta \leqslant C \log (T(h, r))
$$

pour tout réel positif $r \in \mathbb{R} \backslash E$, où $E$ est un ensemble de mesure de Lebesgue finie.

Afin de donner une idée de la démonstration de proposition 4.8, nous rappelons le classique lemme de la dérivée logarithmique de Nevanlinna, voir [Nev29].

ThÉorème 4.12. Soit $\tau: \mathbb{C} \rightarrow \mathbb{P}^{1}$ une application méromorphe. Alors il existe un ensemble $E \subset \mathbb{R}_{+}$ de mesure de Lebesgue finie, tel que pour tout $r \in \mathbb{R} \backslash E$ on ait

$$
\int_{0}^{2 \pi} \log ^{+}\left|\frac{\tau^{\prime}(r \exp (i \theta))}{\tau(r \exp (i \theta))}\right| d \theta \leqslant C \log (T(h, r)) .
$$

La proposition 4.8 est une conséquence assez immediate du théorème précédent. En effet, on peut supposer le fibré $D$ très ample, quitte à lui ajouter un multiple assez grand de $A$. Il existe donc des sections $\left(\sigma_{j}\right)_{j=1, \ldots, N}$ de $D$ telles qu'au voisinage de tout point $x \in B$, on puisse extraire de l'ensemble $\log \left(\sigma_{j} / \sigma_{k}\right)_{j, k=1, \ldots, N}$ des coordonnées locales holomorphes.

Considérons maintenant une courbe entière $h_{1}: \mathbb{C} \rightarrow \mathbb{P}^{1}$. Le théorème de Nevanlinna peut être réformulé comme suit : la moyenne sur les cercles du logarithme de $\left|h_{1}^{*}(d z / z) / d t\right|$ est dominée par $\mathcal{O}\left(\log T\left(h_{1}, r\right)\right)$. Enfin, la fonction $t \rightarrow\left|\omega\left(h^{\prime}(t)\right)\right|_{\psi^{-1}}$ s'exprime localement sous la forme : $\mathcal{P}\left(h(t),(d / d t)\left(\log h_{j k}(t)\right)\right)$, où $\mathcal{P}$ est un polynôme, et $h_{j k}:=\sigma_{j} / \sigma_{k} \circ h$. La compacité de $X$ et le lemme de la dérivée logarithmique impliquent alors le résultat (proposition 4.8) ; pour plus de détails, voir [Ru01, pp. 289-302].

Maintenant, si par l'absurde $\omega\left(h^{\prime}\right)$ n'est pas identiquement nulle, on a :

$$
i \partial \bar{\partial} \log \left|\omega\left(h^{\prime}\right)\right|_{\psi^{-1}}^{2} \geqslant h^{*} \Theta_{\psi}(A)
$$

par l'équation de Poincaré-Lelong. Si on intègre l'inégalité ci-dessus à la manière de Nevanlinna, on obtient

$$
\int_{0}^{r} \frac{d t}{t} \int_{D(t)} i \partial \bar{\partial} \log \left|\omega\left(h^{\prime}\right)\right|_{\psi^{-1}}^{2} \geqslant T_{A}(h, r)
$$

pour tout réel positif $r$. La formule de Jensen donne ensuite

$$
\int_{0}^{2 \pi} \log ^{+}\left|\omega\left(h^{\prime}(r \exp (i \theta))\right)\right|_{\psi^{-1}} d \theta \geqslant T_{A}(h, r)+\mathcal{O}(1),
$$

et compte tenu du fait que notre opérateur $\omega$ est (en particulier) à pôles logarithmiques le long de $D$, le résultat de Wong combiné avec l'inegalité précedente donnent

$$
\log \left(T_{A}(h, r)\right) \geqslant T_{A}(h, r)+\mathcal{O}(1)
$$

pour tout $r \in \mathbb{R} \backslash E$. Lorsque $r \rightarrow \infty$, on obtient une contradiction, et la proposition 4.11 est ainsi démontré.

On se place maintenant à nouveau dans la situation $\left(B, D, N, F, D_{0}, m\right)$ et les hypothèses du corollaire 4.4 . 


\section{F. Campana et M. PăUN}

Proposition 4.13. Nous avons alors les propriétés suivantes :

(1) Il existe un sous-ensemble fini $G=G(F)$ de points $\left(p_{j}\right)_{j \in J}$ de la courbe $D=D_{0}$, tels que pour toute courbe entière $h: \mathbb{C} \rightarrow B$ m-tangente à $D$, l'intersection (ensembliste) de l'image de $h$ avec $D$ est contenue dans la réunion finie $G=G(F) \operatorname{des}\left(p_{j}\right)$.

(2) L'ensemble $G(F)$ est indépendant de $F$.

Ceci acquis, la preuve sera finie, car on peut reprendre les arguments utilisés dans le cadre algébrique : la construction présentée dans le $\S 3$ montre qu'il existe une surface de type général $S^{\prime}$, telle que toute courbe (algébrique ou non) dans $B, m$-tangente à $D$, parametrée par $\mathbb{C}$, et dont l'image ne contient aucun des points $\left(p_{j}\right)$ se relève à $S^{\prime}$.

Mais l'inégalité $\left(c_{1}^{2}-c_{2}\right)>0$ sur les classes de Chern de $S^{\prime}$, et le théorème de McQuillan [McQ98] montrent que l'image de $h$ est contenue dans une courbe algébrique. Les résultats du paragraphe précedent achèvent alors la démonstration, puisque la composée $\varphi \circ h: \mathbb{C} \rightarrow X$ est $m$-tangente à $D$, pour toute application holomorphe $h: \mathbb{C} \rightarrow X$.

Démonstration. Pour montrer l'existence des points $\left(p_{j}\right)$ dans la proposition 4.13(1), observons tout d'abord que $\omega$ peut être vue comme une section méromorphe de $\mathcal{O}_{P}(m q) \otimes A^{-1}$, où $P:=\mathbb{P}\left(T_{B}\right)$.

Supposons que l'image de la courbe $h$ ne soit pas contenue dans $D$. Alors la proposition 4.11 montre que l'image de la courbe dérivée $h^{\prime}: \mathbb{C} \rightarrow \mathbb{P}\left(T_{B}\right)$ est contenue dans une composante irréductible $Y$ de l'ensemble des zéros de $\omega$. On analyse les deux cas qui peuvent apparaitre.

(1) La dimension de la projection $\pi(Y)$ sur $X$ est 1 ; alors en particulier, l'image de $h$ est contenue dans une courbe algébrique, cas traité auparavant.

(2) Si, enfin, $\pi_{Y}: Y \rightarrow B$ est une application (génériquement) finie, il existe un nombre fini de points de $B$ pour lesquels la fibre de $\pi_{\mid Y}$ est de dimension positive. De plus, sur $Y$ (ou plutôt, sa désingularisée), on a un feuilletage canonique $\mathcal{F}$, dont les disques tangents sont décrits comme suit : soit $\tau:(\mathbb{C}, 0) \rightarrow B$ un germe de disque holomorphe, dont l'image de la dérivée projectivisée $\tau^{\prime}$ est contenue dans $Y$ (autrement dit, $\tau$ est tangente au multi-feuilletage définit par $Y$ ). Alors la dérivée de $\tau^{\prime}$ à l'origine est tangente à $\mathcal{F}$.

Il existe encore deux sous-possibilités. On note $\hat{D}$ la rélevée canonique de la courbe $D$ dans $\mathbb{P}\left(T_{Y}\right)$.

Si $\hat{D}$ est contenue dans $Y$, c'est une courbe invariante de $\mathcal{F}$, et donc $h^{\prime}$ ne peut intersecter $\hat{D}$ qu'aux points singuliers de $\mathcal{F}$ (sinon, $h^{\prime}$ est contenue dans $\hat{D}$, par le théorème d'unicité des solutions pour les équations différentielles ordinaires).

Dans l'autre cas, $\hat{D}$ intersecte $Y$ en un nombre fini des points.

Dans les deux cas, les points $\left(p_{j}\right) \subset D$ sont obtenus par projection de $P$ sur $B$, soit des singularités de $\mathcal{F}$, soit de l'intersection de la relevée canonique de $D$ avec $Y$. Ce qui achève la preuve de la proposition 4.13(1).

Remarque 4.14. La preuve de la proposition 4.11 fonctionne sans changement notable dans le cadre plus naturel des courbes entières $m$-tangentes à $D$ au sens non-classique. En effet, il suffit d'observer que dans ce cas $\omega\left(h^{\prime}\right)$ est bien définie seulement en dehors du support du diviseur $\sum \nu_{j} \delta_{z_{j}}$ (on emploie ici les notations du début de cette section), et de plus la norme de cette section est bornée. On invoque ensuite le théorème de prolongement des fonctions psh ('plurisousharmoniques'), et le reste des arguments est identique.

\section{Seconde étape : $G(F)$ ne dépend pas de $F$}

Démonstration (suite). On va établir l'indépendance des points $\left(p_{j}\right)$ par rapport à la configuration $F$ utilisée pour construire par éclatements la surface $B_{F}$, et établir ainsi proposition $4.13(2)$. 


\section{VARIÉTÉS FAIBLEMENT SPÉCIALES À COURBES ENTIÈRES DÉGÉNÉRÉES}

La démonstration va aussi se faire en deux étapes : dans la première (lemme 4.15), on montre que la conclusion est vérifiée si la multiplicité du courant d'Ahlfors sur $B$ associé à $h$ a une multiplicité nulle aux points d'intersection de $h(\mathbb{C})$ avec $D$. Dans la seconde (lemme 4.16), on établit cette propriété comme conséquence du fait que la dimension de Kodaira de $B$ est 1 .

Pour ceci, on a besoin de faire quelque rappels concernant les courants associés aux courbes entières, et leurs propriétés numériques. Les références principales qu'on utilisera sont [McQ98, Bru99] et [Pau03].

Soit $X$ une variété projective, et soit $A \rightarrow X$ un fibré ample, muni d'une métrique $\psi$ dont la forme de courbure $\Theta_{\psi}(A)$ est définie positive sur $X$. Si $h: \mathbb{C} \rightarrow X$ est une courbe entière, alors l'indicatrice de croissance de $h$ par rapport à $\Theta_{\psi}(A)$ est définie par

$$
T_{A}(h, r):=\int_{0}^{r} \frac{d t}{t} \int_{\Delta(t)} h^{*} \Theta_{\psi}(A)
$$

où $\Delta(t) \subset \mathbb{C}$ est le disque de rayon $t$. McQuillan associe à $h$ un courant positif fermé de la façon suivante : pour tout réel positif $r$, considérons le courant de type $(n-1, n-1)$ défini par

$$
\left(T_{r}[h], \alpha\right):=\frac{\int_{0}^{r}(d t / t) \int_{\Delta(t)} h^{*} \alpha}{T(h, r)},
$$

où $\alpha$ est une forme de type $(1,1)$, de classe $\mathcal{C}^{\infty}$ sur $X$. Maintenant, si $\left(r_{k}\right)$ est une suite qui tend vers l'infini, la famille de courants $T_{r_{k}}[h]$ est de masse uniformément bornée, donc par précompacité, admet une limite faible, notée $T[h]$. C'est un courant positif, et de plus, quitte à bien choisir la suite $\left(r_{k}\right)$, il sera fermé (cette affirmation est une conséquence des résultats classiques d'Ahlfors et Nevanlinna). On rappelle très brièvement les propriétés numériques de ces courants qui vont servir par la suite.

(1) Si la courbe $h$ est Zariski dense, et si $\{\alpha\}$ est une classe contenant un courant positif fermé, alors $(T[h], \alpha) \geqslant 0$ (voir [McQ98, Pau03]).

(2) On définit la multiplicité asymptotique mult $(T[h], x)$ de la courbe $h$ au point $x \in X$ comme suit : soit $\hat{X}$ l'éclatement de $X$ en $x$, et soit $\hat{h}$ la relevée de la courbe $h$. Si $E$ désigne le diviseur exceptionnel, alors la multiplicité de $h$ en $x$ est par définition mult $(T[h], x):=\left(T[\hat{h}], c_{1}(E)\right)$. Le résultat dont nous aurons besoin est le suivant : si h est une courbe entière Zariski dense tracée sur une surface projective, et dont la multiplicité asymptotique en un point est positive, alors la classe de cohomologie du courant $T[h]$ contient un courant kählérien (voir [McQ05] et également [Pau03]). Le résultat analogue en géométrie algébrique est le suivant : soit $C$ une courbe nef sur une surface projective $S$, et soit $x \in C$; on suppose que la transformée propre $\hat{C}$ de $C$ par rapport à l'éclatement de $S$ en $x$ est d'autointersection positive. Alors la courbe $C$ est 'big' (lorsque vue comme diviseur sur $S$ ).

Après ce bref détour, revenons aux ensembles $F \subset B_{0}$, et aux surfaces $B_{F}$ correspondantes. On fixe une de ces surfaces $B=B_{F_{0}}$ associée à $F_{0}$ comme surface de référence, et à toute courbe $h_{F}: \mathbb{C} \rightarrow B_{F}$, on associe la courbe $h_{F, 0}: \mathbb{C} \rightarrow B$ en projettant $h_{F}$ sur $B_{0}$, et en considérant la transformée propre de cette projection sur $B_{F_{0}}$. On remarquera que si la courbe $h_{F}$ est $m$-tangente à $D_{F}$, alors la courbe $h_{F, 0}$ est $m$-tangente à $D_{F_{0}}$, sauf (éventuellement) en un nombre fini de points contenus dans $F \cup F_{0}$. On utilise cette observation pour montrer le lemme suivant (qui renforce Lemme 4.10). Dans l'enoncé suivant, on emploie les notations et hypothèses du debut du $\S 4.4$.

Lemme 4.15. Soit $F \subset D_{0}$ une configuration, et soit $h_{F}: \mathbb{C} \rightarrow B_{F}$ une courbe entière Zariski dense, telle que mult $\left(T\left[h_{F, 0}\right], x\right)=0$, en tout point $x \in B$. Alors la section méromorphe $\omega\left(h_{F, 0}^{\prime}\right)$ du fibré $h_{F, 0}^{*} A^{-1}$ s'annule identiquement sur $\mathbb{C}$. 


\section{F. Campana et M. PăUN}

Démonstration. La démonstration est identique à celle du lemme 4.10 ; la non-annulation de la section méromorphe $\omega\left(h_{F, 0}^{\prime}\right)$ et l'équation de Poincaré-Lelong montrent que

$$
i \partial \bar{\partial} \log \left|\omega\left(h_{F, 0}^{\prime}\right)\right|_{\psi^{-1}}^{2}+N \sum_{p} \sum_{j} \nu_{j, p} \delta_{z_{j, p}} \geqslant h_{F, 0}^{*} \Theta_{\psi}(A)
$$

où l'on a noté $\left(s_{p}\right)_{p=1, \ldots, M}$ l'ensemble fini des points du diviseur $D$ ou $h_{F, 0}$ n'est pas $m$-tangente à $D$, et les quantités intervenant dans la seconde somme sont définies par $h_{F, 0}^{-1} s_{p}=\sum_{j} \nu_{j, p} z_{j, p}$. En intégrant cette inégalité, on obtient :

$$
\int_{0}^{r} \frac{d t}{t} \int_{D(t)} i \partial \bar{\partial} \log \left|\omega\left(h_{F, 0}^{\prime}\right)\right|_{\psi^{-1}}^{2}+N \sum_{p=1}^{M} \sum_{0<\left|z_{j, p}\right|<r} \nu_{j, p} \log \frac{r}{\left|z_{j, p}\right|} \geqslant T_{A}\left(h_{F, 0}, r\right)
$$

pour tout réel positif $r$. La formule de Jensen donne ensuite

$$
\int_{0}^{2 \pi} \log ^{+}\left|\omega\left(h^{\prime}(r \exp (i \theta))\right)\right|_{\psi^{-1}} d \theta+N \sum_{p} \sum_{0<\left|z_{j, p}\right|<r} \nu_{j, p} \log \frac{r}{\left|z_{j, p}\right|} \geqslant T_{A}\left(h_{F, 0}, r\right)+\mathcal{O}(1) .
$$

On divise l'inegalité précédente par l'indicatrice de croissance $T_{A}\left(h_{F, 0}, r\right)$, et on fait $r \rightarrow \infty$; on en déduit

$$
1<N \sum_{p=1}^{M} \operatorname{mult}\left(T\left[h_{F, 0}\right], s_{p}\right)
$$

Mais par hypothèse, la multiplicité de la courbe en un point arbitraire vaut zéro, et la contradiction ainsi obtenue démontre la lemme.

On remarquera ici que la forme $\omega$ est indépendante de $F$.

L'étape suivante de la preuve est de montrer qu'on a mult $\left(T\left[h_{F, 0}\right], x\right)=0$, pour tout $x \in B$, si $h_{F, 0}$ est Zariski dense.

C'est une conséquence du résultat général suivant.

Lemme 4.16. Soit $B$ une surface projective non-singulière, et soit $h: \mathbb{C} \rightarrow B$ une courbe entière Zariski dense, tangente à un multi-feuilletage. Si mult $(T[h], x)>0$ pour un point $x \in B$, alors $\kappa(B) \leqslant 0$.

Démonstration. Cet enoncé est implicite dans les articles [McQ98, Bru99], mais pour la commodité du lecteur, on va en donner la démonstration.

Par hypothèse, l'image de la courbe dérivée $h^{\prime}$ de $h$ est contenue dans un diviseur $Y \subset \mathbb{P}\left(T_{B}\right)$, et $h^{\prime}$ est tangente à un feuilletage sur $Y$.

Quitte à modifier $Y$, on peut supposer qu'on se trouve dans la situation suivante : il existe $\pi: B_{1} \rightarrow B$ une application surjective (composée d'une application finie avec une suite d'éclatements), et une courbe $h_{1}: \mathbb{C} \rightarrow B_{1}$, Zariski dense, tangente à un feuilletage réduit au sens de Seidenberg (voir, e.g., [McQ98] et les références dans cet article), et telle que $\pi \circ h_{1}=h$. Dans ce cas, les résultats de McQuillan et Brunella [McQ98, Bru99] montrent que

$$
\left(T\left[h_{1}\right], K_{B_{1}}\right) \leqslant 0 .
$$

Par ailleurs, on a $K_{B_{1}}=\pi^{*} K_{B}+R$, où $R$ est un diviseur effectif, et la propriété (1) citée auparavant montre que $\left(T\left[h_{1}\right], c_{1}(R)\right) \geqslant 0$; on en déduit ainsi l'inégalité :

$$
\left(T\left[h_{1}\right], \pi^{*} K_{B}\right) \leqslant 0 .
$$




\section{VARIÉTÉS FAIBLEMENT SPÉCIALES À COURBES ENTIÈRES DÉGÉNÉRÉES}

Supposons à présent que $\kappa(B) \geqslant 1$. Alors le diviseur canonique admet une décomposition de Zariski $K_{B}=P+E$, où $P$ est un diviseur nef, de dimension numérique au moins 1 , et $E$ est un diviseur effectif. La relation précédente montre que $(T[h], P) \leqslant 0$ (car l'image directe du courant $T\left[h_{1}\right]$ par $\pi$ est simplement $T[h]$ ). Par hypothèse, la courbe $h$ admet une multiplicité positive en $x$, et la propriété $(2)$ montre que $(T[h], P)>0$. Le lemme est démontré.

En conclusion des résultats précédents, il existe une section $\omega \in H^{0}\left(B,\left[\operatorname{Sym}^{N}\right]\left(\Omega_{(B / \Delta)}^{1}\right) \otimes A^{-1}\right)$ telle que pour tout ensemble $F$, et toute courbe entière $h_{F}: \mathbb{C} \rightarrow B_{F}$ Zariski dense et $m$-tangente à $D_{F}$, on ait $\omega\left(h_{F, 0}^{\prime}\right)=0$.

Donc il existe un ensemble fini $G$ de points $\left(p_{j}\right)$ tel que pour tout ensemble $F$, l'intersection de la projection des courbes entières Zariski denses de $X_{F}$ sur $B_{F}$ est contenue dans la réunion des $\left(p_{j}\right)$. La proposition 4.13 , et donc aussi le théorème 4.3 sont ainsi complètement démontrés.

\section{REFERENCES}

Bog77 F. Bogomolov, Families of curves on surfaces of general type, Sov. Math. Dokl. 18 (1977), 1294-1297.

BT03 F. Bogomolov and Y. Tschinkel, Special elliptic fibrations, in Proc. Fano Conf., Torino, 2003, ed. A. Conte, math.AG/0303044.

Bru99 M. Brunella, Courbes entires et feuilletages holomorphes, Enseign. Math. (2) 45 (1999), 195-216.

Cam04 F. Campana, Special varieties, orbifolds and classification theory, Ann. Inst. Fourier (Grenoble) $\mathbf{5 4}$ (2004), 499-665.

Cam05 F. Campana, Fibres multiples sur les surfaces : aspects hyperboliques et arithmétiques, Manuscripta Math. 117 (2005), 429-461.

Dem97 J.-P. Demailly, Variétés hyperboliques et équations différentielles algébriques, Gaz. Math. 73 (1997), $3-23$.

Des77 M. Deschamps, Courbes de genre géométrique borné sur une surface de type général, Séminaire Bourbaki 1977/78, exp. 519, Lecture Notes in Mathematics, vol. 710 (Springer, Berlin, 1977), 233-247.

FM94 R. Friedman and J. Morgan, Smooth four-manifolds and complex surfaces (Springer, Berlin, 1994).

GK73 P. Griffiths and J. King, Nevanlinna theory and holomorphic maps between algebraic varieties, Acta Math. 130 (1973), 145-220.

HT00 J. Harris and Y. Tschinkel, Rational points on quartics, Duke Math. J. 104 (2000), 477-500.

McQ98 M. McQuillan, Diophantine approximations and foliations, Publ. Math. Inst. Hautes Études Sci. 87 (1998), 121-174.

McQ05 M. McQuillan, Uniform uniformisation, Preprint, Institut des Hautes Études Scientifiques (2005).

Nev29 R. Nevanlinna, Le théorème de Picard-Borel et la théorie des fonctions méromorphes (GauthierVillars, Paris, 1929).

Pau03 M. Păun, Currents associated to transcendental entire curves on compact Kähler manifolds, Preprint (2003).

Ru01 M. Ru, Nevanlinna theory and its relations to Diophantine approximation (World Scientific, Singapore, 2001).

Siu97 Y. T. Siu, personal communication to J.-P. Demailly (see [Dem97]).

Won99 P. M. Wong, Nevanlinna theory for holomorphic curves in projective varieties, Preprint (1999).

Frédéric Campana campana@iecn.u-nancy.fr

Département de Mathématiques, Université de Nancy, F-54506 Vandoeuvre-les-Nancy, France

Mihai Păun mihai.paun@iecn.u-nancy.fr

Département de Mathématiques, Université de Nancy, F-54506 Vandoeuvre-les-Nancy, France 